\title{
A QoE-Aware Scheduler for OFDMA Networks
}

\author{
Victor F. Monteiro, Diego A. Sousa, Tarcisio F. Maciel, \\ Francisco Rafael M. Lima, and Francisco Rodrigo P. Cavalcanti
}

\begin{abstract}
In this paper, we present a Quality of Experience (QoE)-aware scheduler aiming at maximizing the minimum Mean Opinion Score (MOS) in a system subject to attaining at least a given number of satisfied users. Different from the benchmark solution, the proposed one takes into account all the data that the User Equipments (UEs) have already received, and not only the data of a specific instant of time, thus suitably excluding UEs already satisfied. According to simulation results, the proposed solution not only improves the users' satisfaction and the minimum experienced MOS in the system, but it also doubles the capacity in terms of supported number of users compared to benchmark solution. It is also able to handle imperfections on the Channel State Information (CSI).
\end{abstract}

Index Terms-Quality of Experience, Minimal Mean Opinion Score Maximization, Radio Resource Allocation.

\section{INTRODUCTION}

The next generation of wireless communications, the $5^{\text {th }}$ Generation (5G), is expected to support tens or even hundreds of billions of devices as a result of new applications beyond personal communication [2]. Due to this diversity of devices, the experience perceived by the users may differ, even if they are subjected to similar Quality of Service (QoS) scenarios, i.e., same data rates, latency, etc.

Therefore, the conventional network-centric view of the system will no longer be enough, needing to be replaced by user-centric approaches. These new approaches should adopt Quality of Experience (QoE) metrics, which consider the subjective perception of the end user, instead of QoS metrics, which are typically network oriented. QoE is generally evaluated by a Mean Opinion Score (MOS) ranging from 1 to 5 [3].

One possibility to provide better user satisfaction is to consider QoE-aware schedulers to manage the limited radio resources. In [4], a power allocation scheme is proposed targeting at maximizing QoE for video transmissions over Multiple Input Multiple Output (MIMO) systems. The problem is decomposed into sub-problems and a bisection search algorithm is used to obtain their optimal solutions. In [5], a multi-cell coordination among multiple Base Stations

The Associate Editors coordinating the review of this manuscript and approving it for publication were Prof. Cecílio José Lins Pimentel and Prof. Marcelo da Silva Pinho.

The authors are with the Wireless Telecommunications Research Group (GTEL), Federal University of Ceará (UFC), Fortaleza, Ceará, Brazil.

Diego A. Sousa is also with Federal Institute of Education, Science, and Technology of Ceará, Acaraú, Brazil. Tarcisio F. Maciel and Fco. Rodrigo P. Cavalcanti are also with Department of Teleinformatics Engineering, UFC. Fco. Rafael M. Lima is also with Department of Computer Engineering, UFC.

A preliminary version of this paper was presented in XXXIII Simpósio Brasileiro de Telecomunicações (SBrT'15), Juiz de Fora, MG, Brazil September 1-4, 2015. [1]

Digital Object Identifier: 10.14209/jcis.2016.3
(BSs) is investigated for interference mitigation and overall QoE maximization. The problem is formulated as a local cooperative game, where BSs are encouraged to cooperate with their peer nodes in the adjacent cells when scheduling users and allocating power.

Opportunistic algorithms, such as the algorithms proposed in [4] and [5], which try to maximize the sum of QoE of the users in a system may penalize users with poor link conditions. To overcome this problem, the solution proposed in [6] firstly allocates subcarriers to all the users in order to guarantee their minimal transmit rate requirement, then the remaining subcarriers are allocated to the users who can achieve the best QoE gain. In [7], a Proportional Fair algorithm scheduling is proposed considering not only the users' QoE maximization, but also the fairness among users.

Another strategy to increase the fairness between the users is to maximize the minimum MOS, as done in [8]. The authors formulate a problem aiming at maximizing the minimum MOS of the users in a system subject to attaining at least a given number of satisfied users. Therein, an algorithm for Resource Block (RB) assignment and power allocation is proposed to solve this problem. The presented results showed that the proposed solution outperforms state-of-the-art algorithms, as well as it performs close to the optimal solution.

The present work proposes a step ahead with respect to [8]. The solution presented in [8] does not take into account the time dimension, trying to satisfy the users at each Transmission Time Interval (TTI), independently of previous allocations. Therefore, considering the time dimension, this solution is not as fair as it should be, since some users can be satisfied for longer periods of time than others, mainly when there is a high number of users in the system competing for a limited resources. This results in a capacity problem where the system cannot deal with its high number of users.

To overcome the previous cited issue of [8], this work presents a RB scheduler considering that the users should be satisfied on average over a certain timespan, thus admitting that some users can be unsatisfied at some particular TTI. Thus, different from [8], when estimating the users' MOS in an instant of time, the proposed solution takes into account all the data that they have already received, and not only the data of the current TTI. Furthermore, when a user is already satisfied at a given TTI, he or she will not be considered during the resource scheduling for that TTI.

The remainder of this paper is organized as follows. Sections II and III introduce the network model and the problem formulation, respectively. Sections IV and V present the optimal and the proposed solutions, respectively. The performance of the involved algorithms is evaluated via computational simulations in Section VI. Finally, in 
Section VII, the main conclusions of our work are presented.

\section{SySTEM MODELING}

We consider a downlink single-cell Orthogonal Frequency Division Multiple Access (OFDMA) environment mainly based on Long Term Evolution (LTE) specifications [9], [10] and in which an Evolved Node B (eNB) serves a subset $\mathcal{U}$ of $U$ User Equipments (UEs). Due to the diversity of applications with distinct requirements, we consider that the UEs are separated into different mobile subscription plans. For example, there is a priority plan with stricter requirements for emergency services, as fire brigade, police and ambulances, and another one for UEs in general. We define $\mathcal{S}=\{1,2, \ldots, S\}$ as the set of subscription plans and $\mathcal{U}_{s}$ as the set of subscribers of service plan $s \in \mathcal{S}$, in which $\bigcup_{s \in \mathcal{S}} \mathcal{U}_{s}=\mathcal{U}$. Moreover, we consider that each user subscribes only a single service plan, i.e., $\bigcap_{s \in \mathcal{S}} \mathcal{U}_{s}=\emptyset$.

We consider a RB as the minimum allocable time-frequency block in the considered LTE system. Each RB is composed of a number of adjacent subcarriers in frequency domain and of a number of OFDMA symbols spanning the duration of one TTI in time domain. Moreover, we assume that the eNB manages the allocation of a set $\mathcal{K}$ of $K$ RBs to the UEs. We also assume that each UE and the eNB is equipped with a single antenna and the total power of the eNB is equal to $P_{\mathrm{t}}$, which is equally distributed among all RBs. Thus, the power $p_{u, k}$ used by the eNB to transmit to UE $u$ through the RB $k$ is $p_{u, k}=P_{\mathrm{t}} / K$.

The channel coefficient $h_{u, k}[t]$ between the eNB and the UE $u$ in TTI $t$ is approximated by the coefficient of the first symbol of the mid subcarrier that composes the RB. Moreover, we assume that it remains constant during the period of one TTI.

In practice, the channel is estimated by the UE using pilot symbols transmitted by the eNB. In order to simulate a more realistic scenario, we consider that the channel estimate received by the eNB, $\hat{h}_{u, k}[t]$, corresponds to the real channel measure delayed by $\Delta t$ TTIs:

$$
\hat{h}_{u, k}[t]=h_{u, k}[t-\Delta t] .
$$

Therefore, the Signal to Noise Ratio (SNR) information related to $\mathrm{UE} u$ on $\mathrm{RB} k$ and available in the eNB is given by

$$
\gamma_{u, k}[t]=\frac{p_{u, k}\left|\hat{h}_{u, k}[t]\right|^{2}}{\sigma^{2}},
$$

in which $\sigma^{2}$ denotes the thermal noise power.

We assume that the data transmission considers a link adaptation scheme that allows the eNB to transmit with a set of $M$ possible Modulation and Coding Schemes (MCSs) with very low BLock Error Rate (BLER). The MCS selected by the eNB is a function of the SNR $\gamma_{u, k}[t]$.

The total data transmitted to UE $u$ in TTI $t$ is given by

$$
R_{u}[t]=\sum_{k=1}^{K} r_{u, k}[t] x_{u, k}[t],
$$

in which $r_{u, k}[t]$ is the achievable transmitted rate by the eNB to UE $u$ on RB $k$ in TTI $t$ and $x_{u, k}[t]$ is the assignment index indicating whether the RB $k$ is allocated to UE $u$ in TTI $t$.

Finally, defining $\phi(\cdot)$ as a function mapping data rate into a MOS value, the QoE $\tau_{u}[t]$ of a UE $u$ at TTI $t$ is given by

$$
\tau_{u}[t]=\phi\left(\frac{\sum_{j=1}^{t} R_{u}[j]}{t}\right) .
$$

\section{PRoblem Formulation}

As previously mentioned, QoE-aware schedulers have been considered as an user-centric approach to manage the limited resources in wireless networks. In this context, we aim at maximizing the minimum MOS of the system in the end of a timespan of $T$ TTIs by assigning RBs to the UEs being served by an eNB.

Furthermore, this work considers that at least $\varphi_{s}$ UEs should be satisfied for each service plan $s$ in the end of a timespan, i.e., for each service plan $s$, at least $\varphi_{s}$ UEs should have a MOS equal to or higher than a given target MOS value $n_{s}$ in the end of a timespan. Considering $U_{s}$ as the number of UEs of service plan $s$, the ratio $\alpha_{s}=\frac{\varphi_{s}}{U_{s}}$ is defined as the satisfaction factor of service plan $s$.

The studied problem can now be summarized as follows:

$$
\begin{array}{ll}
\operatorname{maximize} & \min _{u \in \mathcal{U}}\left\{\tau_{u}[T]\right\} \\
\text { subject to } & \sum_{u=1}^{U_{s}} \delta\left(\tau_{u}[T], n_{s}\right) \geq \varphi_{s}, \forall s \in \mathcal{S} \\
& \sum_{u=1}^{U} x_{u, k}[t] \leq 1, \forall k \in \mathcal{K}, \forall t \in[1, T] \\
& x_{u, k}[t] \in\{0,1\}, \forall u \in \mathcal{U}, \forall k \in \mathcal{K}, \forall t \in[1, T],
\end{array}
$$

in which $\delta(a, b)$ represents the step function given by

$$
\delta(a, b)= \begin{cases}1, & \text { if } a \geq b, \\ 0, & \text { if } a<b .\end{cases}
$$

The objective function $\min _{u \in \mathcal{U}}\left\{\tau_{u}[T]\right\}$ being maximized in $(5 \mathrm{a})$ represents the system minimum MOS in the end of a timespan of $T$ TTIs. The inequality in (5b) imposes that at least $\varphi_{s}$ out of the $U_{s}$ UEs of service plan $s$ have MOS equal to or greater than $n_{s}$. Finally, the last two constraints, (5c) and (5d), assure that a RB will be allocated to only one UE at a time.

The optimal solution of (5) cannot be obtained in real systems, since it should be necessary to know a priori the values of $h_{u, k}[t]$ during the whole timespan to decide which would be the best TTIs to allocate each resource to each UE. This kind of information is clearly not available, since it is not possible to precisely forecast the future channel state. Even though, in the next section we develop the optimal solution as a linear optimization problem to be used as a benchmark solution. 


\section{Optimal SOLUtion}

In this section, (5) is reformulated as a Mixed Integer Linear Programming (MILP), which can be solved by standard algorithms, such as the Branch and Bound (BB) method [11]. Otherwise, it would be necessary to use the brute force or exhaustive search to solve (5).

The non-linear objective in (5a) can be linearized by introducing a slack variable and new constraints as follows:

$$
\begin{array}{ll}
\operatorname{maximize} & \lambda, \\
\text { subject to } & \tau_{u}[T] \geq \lambda, \forall u \in \mathcal{U} .
\end{array}
$$

Considering $\phi(\cdot)$ as a strictly increasing function, then there is an inverse function $\phi^{-1}(\cdot)$ mapping the possible MOS values of the UEs into corresponding required rate values [12]. Therefore, we can define $\mu=\phi^{-1}(\lambda)$ and $\psi_{u}=\phi^{-1}\left(n_{s}\right), \forall u \in \mathcal{U}_{s}$ and $\forall s \in \mathcal{S}$, in which $\psi_{u}$ is the required transmit rate for UE $u$ to be satisfied.

Now, considering the previous definitions and replacing (3) and (4) in (7b), we have:

$$
\begin{aligned}
& \phi\left(\frac{1}{T} \sum_{k=1}^{K} \sum_{t=1}^{T} r_{u, k}[t] x_{u, k}[t]\right) \geq \lambda, \forall u \in \mathcal{U} \Rightarrow \\
& \frac{1}{T} \sum_{k=1}^{K} \sum_{t=1}^{T} r_{u, k}[t] x_{u, k}[t] \geq \mu, \forall u \in \mathcal{U} .
\end{aligned}
$$

Similarly, (5b) can be replaced by:

$$
\sum_{u=1}^{U_{s}} \delta\left(\frac{1}{T} \sum_{k=1}^{K} \sum_{t=1}^{T} r_{u, k}[t] x_{u, k}[t], \psi_{u}\right) \geq \varphi_{s}, \forall s \in \mathcal{S} .
$$

Therefore, (5) can be rewritten as:

maximize $\mu$

subject to $\frac{1}{T} \sum_{k=1}^{K} \sum_{t=1}^{T} r_{u, k}[t] x_{u, k}[t] \geq \mu, \forall u \in \mathcal{U}$,

$$
\begin{aligned}
& \sum_{u=1}^{U_{s}} \delta\left(\frac{1}{T} \sum_{k=1}^{K} \sum_{t=1}^{T} r_{u, k}[t] x_{u, k}[t], \psi_{u}\right) \geq \varphi_{s}, \forall s \in \mathcal{S} \\
& \sum_{u=1}^{U} x_{u, k}[t] \leq 1, \forall k \in \mathcal{K}, \forall t \in[1, T] \\
& x_{u, k}[t] \in\{0,1\}, \forall u \in \mathcal{U}, \forall k \in \mathcal{K}, \forall t \in[1, T]
\end{aligned}
$$

We can observe that problem (10) is still non linear due to constraint $(10 \mathrm{c})$. In order to linearize this problem, we introduce two binary operators: $q_{u, s}$, which is equal to 1 if the UE $u$ subscribes the service plan $s$ or 0 otherwise, and $\rho_{u}$, which assumes the value 1 if the UE $u$ is satisfied, $\tau_{u} \geq \phi\left(\psi_{u}\right)$, and 0 otherwise.
Replacing these new operators in (10), we have:

$$
\begin{aligned}
\text { maximize } & \mu \\
\text { subject to } & \frac{1}{T} \sum_{k=1}^{K} \sum_{t=1}^{T} r_{u, k}[t] x_{u, k}[t] \geq \mu, \forall u \in \mathcal{U} \\
& \frac{1}{T} \sum_{k=1}^{K} \sum_{t=1}^{T} r_{u, k}[t] x_{u, k}[t] \geq \psi_{u} \rho_{u}, \forall u \in \mathcal{U}, \\
& \sum_{u=1}^{U} q_{u, s} \rho_{u} \geq \varphi_{s}, \forall s \in \mathcal{S} \\
& \sum_{u=1}^{U} x_{u, k}[t] \leq 1, \forall k \in \mathcal{K}, \forall t \in[1, T] \\
& x_{u, k}[t] \in\{0,1\}, \forall u \in \mathcal{U}, \forall k \in \mathcal{K}, \forall t \in[1, T] .
\end{aligned}
$$

At this point, (5) has already been reformulated as a MILP. Therefore, we will reformulate (11) in a compact form using tensorial notation. For this, we need to introduce some concepts and definitions. The first one is the concept of unfolding. We arrange the elements $x_{u, k}[t]$ in a multi-dimensional array $\mathbf{X} \in \mathbb{R}^{U \times K \times T}$ and we denote $\mathbf{X}^{(2)} \in \mathbb{R}^{K \times U \cdot T}$ as the mode-2 unfolding of $\underline{\mathbf{X}}$, in which the elements $x^{(2)}$ of $\mathbf{X}^{(2)}$ are defined as a function of the elements of $\underline{\mathbf{X}}$ as $x_{k, u+(t-1) U}^{(2)}=x_{u, k}[t]$ [13]. In a similar way, the elements $r_{u, k}[t]$ form the multi-dimensional array $\underline{\mathbf{R}}$, and $\mathbf{R}^{(2)}$ is the mode- 2 unfolding of $\underline{\mathbf{R}}$.

We consider $\mathbf{A} \odot \mathbf{B}$ as the element-wise product between two equal-size matrices, called Hadamard product, and $\mathbf{A} \otimes \mathbf{B}$ as the Kronecker product expressed as

$$
\mathbf{A} \otimes \mathbf{B}=\left[\begin{array}{cccc}
a_{11} \mathbf{B} & a_{12} \mathbf{B} & \cdots & a_{1 J} \mathbf{B} \\
a_{21} \mathbf{B} & a_{22} \mathbf{B} & \cdots & a_{2 J} \mathbf{B} \\
\vdots & \vdots & \ddots & \vdots \\
a_{I 1} \mathbf{B} & a_{I 2} \mathbf{B} & \cdots & a_{I J} \mathbf{B}
\end{array}\right],
$$

in which $\mathbf{A} \in \mathbb{R}^{I \times J}$ and $\mathbf{B} \in \mathbb{R}^{T \times R}$. Finally, we define the vec $\{\cdot\}$ operation as $\operatorname{vec}\{\mathbf{Z}\}=\left[\begin{array}{llll}\mathbf{z}_{1}^{\mathrm{T}} & \mathbf{z}_{2}^{\mathrm{T}} & \ldots & \mathbf{z}_{n}^{\mathrm{T}}\end{array}\right]^{\mathrm{T}}$, in which $\mathbf{z}_{i}$ is the $i$-th column of matrix $\mathbf{Z}$.

To simplify the notation, we rename the following variables: $\mathbf{x}=\operatorname{vec}\left\{\mathbf{X}^{(2)^{\mathrm{T}}}\right\}$ and $\mathbf{r}=\operatorname{vec}\left\{\mathbf{R}^{(2)^{\mathrm{T}}}\right\}$. Arranging the elements $q_{u, s}$ into the matrix $\mathbf{Q}$, we can now rewrite (11) as

maximize $\mu$

subject to $T \mu \cdot \mathbf{1}_{U}-\left[\left(\mathbf{1}_{T K}^{\mathrm{T}} \otimes \mathbf{I}_{U}\right) \odot\left(\mathbf{1}_{U} \otimes \mathbf{r}^{\mathrm{T}}\right)\right] \cdot \mathbf{x} \leq \mathbf{0}_{U}$,

$$
\begin{aligned}
& {\left[\left(T \boldsymbol{\psi} \otimes \mathbf{1}_{U}^{\mathrm{T}}\right) \odot \mathbf{I}_{U}\right] \cdot \boldsymbol{\rho}-} \\
& {\left[\left(\mathbf{1}_{T K}^{\mathrm{T}} \otimes \mathbf{I}_{U}\right) \odot\left(\mathbf{1}_{U} \otimes \mathbf{r}^{\mathrm{T}}\right)\right] \cdot \mathbf{x} \leq \mathbf{0}_{U},} \\
& -\mathbf{Q}^{\mathrm{T}} \boldsymbol{\rho} \leq-\boldsymbol{\varphi} \\
& {\left[\mathbf{I}_{K T} \otimes \mathbf{1}_{U}^{\mathrm{T}}\right] \mathbf{x} \leq \mathbf{1}_{K T},} \\
& \mathbf{x} \text { and } \boldsymbol{\rho} \text { are binary vectors, }
\end{aligned}
$$

in which the elements $\psi_{s}, \rho_{u}$ and $\varphi_{u}$ are respectively arranged into the column vectors $\boldsymbol{\psi}, \rho$ and $\boldsymbol{\varphi}, \mathbf{I}_{U}$ is a $U \times U$ identity 
matrix, $\mathbf{0}_{U}$ is a column vector with $U$ zeros and $\mathbf{1}_{U}$ is a column vector with $U$ ones.

At this point, the variables of our problem are: $\mu, \mathbf{x}$ and $\rho$. To simplify even more the notation, they can be arranged into one single vector $\mathbf{w}$, in which

$$
\mathbf{w}=\left[\mu\left|\mathbf{x}^{\mathrm{T}}\right| \boldsymbol{\rho}^{\mathrm{T}}\right]^{\mathrm{T}} .
$$

Then, using $\mathbf{w}$ in the definition of $\mathbf{a}, \mathbf{B}$ and $\mathbf{C}$ as below

$$
\begin{aligned}
\mathbf{a} & =\left[\begin{array}{l|l|l}
1 & \mathbf{0}_{U T K}^{\mathrm{T}} \mid \mathbf{0}_{U}^{\mathrm{T}}
\end{array}\right]^{\mathrm{T}} \Rightarrow \mathbf{a}^{\mathrm{T}} \mathbf{w}=\mu, \\
\mathbf{B} & =\left[\begin{array}{ll|l}
\mathbf{0}_{U T K}\left|\mathbf{I}_{U T K}\right| \mathbf{0}_{U T K \times U}
\end{array}\right] \Rightarrow \mathbf{B w}=\mathbf{x}, \\
\mathbf{C} & =\left[\begin{array}{ll}
\mathbf{0}_{U \times(1+U T K)} \mid \mathbf{I}_{U}
\end{array}\right] \Rightarrow \mathbf{C w}=\boldsymbol{\rho},
\end{aligned}
$$

we can finally rewrite the optimization problem as

$$
\begin{aligned}
& \operatorname{maximize} \mathbf{a}^{\mathrm{T}} \cdot \mathbf{w}, \\
& \text { subject to } \mathbf{D} \cdot \mathbf{w} \leq \mathbf{e},
\end{aligned}
$$

in which

$$
\mathbf{D}=\left[\begin{array}{c}
T \mathbf{1}_{U} \mathbf{a}-\left[\left(\mathbf{1}_{T K}^{\mathrm{T}} \otimes \mathbf{I}_{U}\right) \odot\left(\mathbf{1}_{U} \otimes \mathbf{r}^{\mathrm{T}}\right)\right] \mathbf{B} \\
{\left[\left(T \boldsymbol{\psi} \otimes \mathbf{1}_{U}^{\mathrm{T}}\right) \odot \mathbf{I}_{U}\right] \cdot \mathbf{C}-\left[\left(\mathbf{1}_{T K}^{\mathrm{T}} \otimes \mathbf{I}_{U}\right) \odot\left(\mathbf{1}_{U} \otimes \mathbf{r}^{\mathrm{T}}\right)\right] \mathbf{B}} \\
-\mathbf{Q}^{\mathrm{T}} \mathbf{C} \\
{\left[\mathbf{I}_{K} \otimes \mathbf{1}_{U T}^{\mathrm{T}}\right] \mathbf{B}}
\end{array}\right]
$$

and,

$$
\mathbf{e}=\left[\mathbf{0}_{U}^{\mathrm{T}}\left|\mathbf{0}_{U}^{\mathrm{T}}\right|-\boldsymbol{\varphi}^{\mathrm{T}} \mid \mathbf{1}_{K}^{\mathrm{T}}\right]^{\mathrm{T}}
$$

\section{QOE-AWARE SCHEDULER FOR IMPROVING SATISFACTION IN OFDMA SYSTEMS}

As already mentioned, the solution of (15) cannot be obtained in real systems, since it requires to know a priori future values of $h_{u, k}[t]$. For that reason, in the following, we propose a heuristic solution.

The proposed Radio Resource Allocation (RRA) algorithm is sketched in the flowchart of Figure 1. It is divided into three parts:

i) satisfied users exclusion, blocks (1) to (3);

ii) minimum number of satisfied users per service satisfaction, blocks (4) to (11); and

iii) minimum MOS maximization, blocks (12) to (16).

The first part consists in estimating the users' MOS taking into account all the data that they have already received, block (2), and excluding the UEs who are satisfied, block (3). In [8], this part was not considered, resulting, sometimes, in the allocation of RBs to UEs which were already satisfied, while leaving others in starvation.

The second part tries to satisfy $\varphi_{s}$ UEs for all service $s \in \mathcal{S}$. It works in a loop, allocating RBs to the UEs with highest transmit rate on each RB, blocks (4) and (5). When someone gets satisfied, he/she is removed, blocks (6) and (7). Besides this, if a service plan $s$ achieves its minimum number of satisfied users, $\varphi_{s}$, all the remaining UEs of this service are also removed, blocks (9) and (10). This loop continues until all the RBs have been allocated, block (8), or all the service plans have achieved their minimum numbers of satisfied UEs, block (11). It is important to note that, when updating the MOS of someone who has been allocated a RB, block (6), the

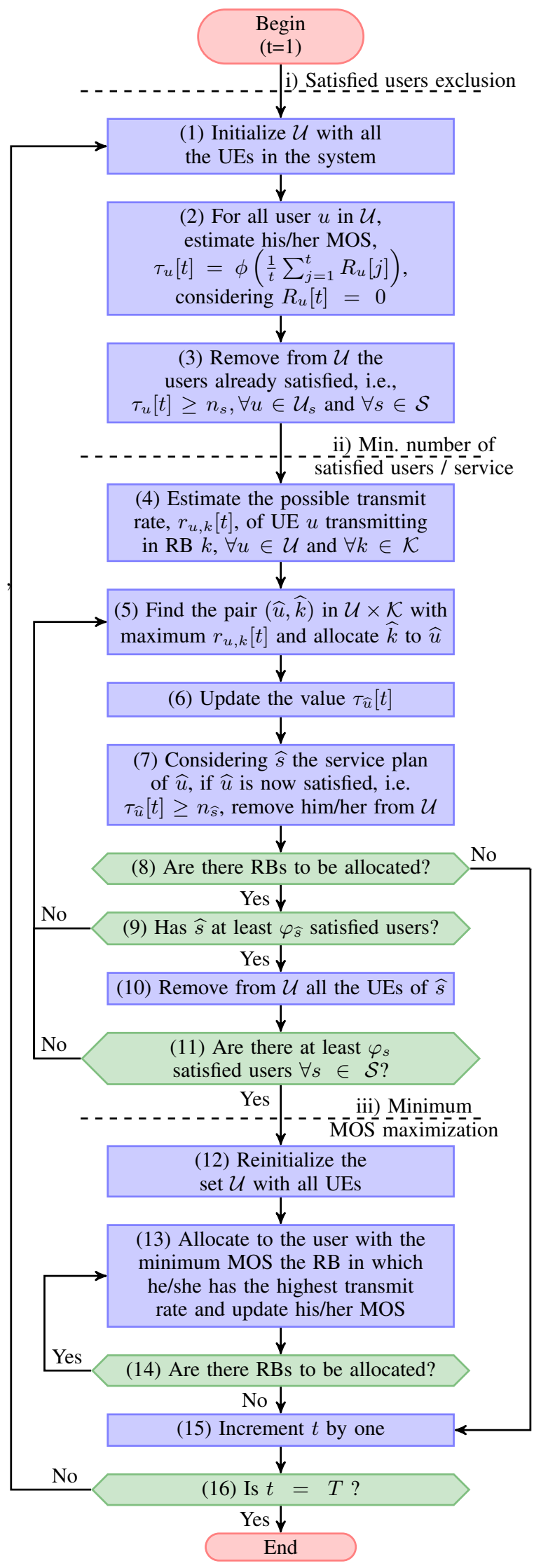

Fig. 1: Flowchart of proposed scheduler. 
time dimension is also considered, since we take into account all the data that have been previously transmitted.

In the last part, the remaining RBs are allocated to the UEs with lowest MOS, in block (13), one at a time, aiming at maximizing the minimum MOS. After the last RB has been allocated, in block (14), the algorithm restarts from block (1), until the end of the timespan, in block (16).

\section{Vi. Performance Evaluation}

In this section, we present the simulation results obtained when comparing the proposed solution with the optimal solution of (15) and with ref. [8], a benchmark algorithm. In Section VI-A and VI-B, the simulation scenario is presented and the results are discussed, respectively.

\section{A. Simulation Assumptions}

The scenario considered in the performance evaluation was aligned with the 3rd Generation Partnership Project (3GPP) LTE architecture [9], [10]. Its description is presented in Section II.

We worked in the carrier frequency of $2 \mathrm{GHz}$ with a $1.4 \mathrm{MHz}$ system bandwidth. Each RB was composed of 12 adjacent subcarriers, resulting in $6 \mathrm{RBs}$ in total. It was considered a small number of RBs due to the high computational effort required to obtain the optimal solution. Besides a small-scale fading, it was also considered as propagation effects a distance-dependent path-loss and a lognormal shadowing component. The value of other parameters are listed in Table I.

The considered MOS function, $\phi(\cdot)$, was proposed in [17] and is given by:

$$
\phi\left(R_{u}\right)=5-\frac{578}{1+\left(\frac{R_{u}+541.1}{45.98}\right)^{2}},
$$

in which $R_{u}$ is the total throughput of UE $u$.

Three different scenarios were adopted to evaluate the performance of the proposed algorithm:

- The first one analyzed the impact of the number of UEs, considering perfect knowledge of the Channel State Information (CSI), i.e., $\Delta t=0$. There was a single service plan, i.e., all the UEs subscribed the same service plan. For this service plan, it was considered a target MOS of 3.5 and satisfaction factor of $100 \%$, i.e., it aimed at satisfying $100 \%$ of the users with a target MOS of at least 3.5.

- The second one also considered $\Delta t=0$. However, different from the first scenario, there were two service plans. Both had a satisfaction factor of $100 \%$, but service plan one considered a target MOS of 3.5 and the other, 4.5. There were 6 UEs in the system, and we analyzed the impact of changing the UEs from service plan 1 to service plan 2 .

- The third scenario analyzed the impact of the delay at CSI estimation, i.e., $\Delta t \geq 0$, in a system with 6 UEs. As the first scenario, there was only one service plan with satisfaction factor of $100 \%$ and target MOS equal to 3.5.

Concerning the benchmark algorithm, [8], different from the present work, it does not consider the time dimension,
Table I: Simulation parameters.

\begin{tabular}{l|l}
\hline \hline \multicolumn{1}{c}{ Parameter } & Value \\
\hline $\begin{array}{l}\text { Maximum eNB transmit power } \\
\left(P_{t}\right)\end{array}$ & $36.8 \mathrm{dBm}[10]$ \\
\hline eNB antenna radiation pattern & Three-sectored [10] \\
\hline Cell radius & $1 \mathrm{~km}$ \\
\hline UE speed $(V)$ & $3 \mathrm{~km} / \mathrm{h} \mathrm{[9]}$ \\
\hline Carrier frequency $\left(f_{c}\right)$ & $2 \mathrm{GHz}[9]$ \\
\hline System bandwidth & $1.4 \mathrm{MHz}[10]$ \\
\hline Subcarrier bandwidth & $15 \mathrm{kHz}$ \\
\hline Number of RBs $(K)$ & 6 \\
\hline Number of subcarriers per RB & 12 \\
\hline Path loss ${ }^{a}$ & $128.1+37.6 \log _{10}(d)$ \\
\hline Antenna gain ${ }^{b}$ & {$[9]$} \\
\hline Downtilt angle & $G_{h}\left(\theta_{h}\right)+G_{v}\left(\theta_{v}\right)[14]$ \\
\hline Log-normal shadowing standard & $8 \mathrm{degrees}$ \\
deviation & $8 \mathrm{~dB}[9]$ \\
\hline Small-scale fading & $3 \mathrm{GPP}$ Typical Urban \\
\hline AWGN power per subcarrier & {$[15]$} \\
\hline Noise figure & $-123.24 \mathrm{dBm}$ \\
\hline Link adaptation & $9 \mathrm{~dB}[9]$ \\
\hline Traffic model & Link level curves \\
\hline Transmission Time Interval & Full buffer \\
\hline Snapshot time span & $1 \mathrm{~ms}$ \\
\hline Number of snapshots & $500 \mathrm{~ms}$ \\
\hline \hline
\end{tabular}

${ }^{a} d$ is the distance from the eNB to the UE in $\mathrm{km}$.

${ }^{b} \theta_{h}$ and $\theta_{v}$ represents the horizontal and vertical angles related to the eNB respectively.

i.e., it does not take into account the previously transmitted data when estimating the users' MOS in (4). Furthermore, it does not have the step of exclusion of satisfied users when allocating the resources, as in Figure 1.

Three metrics were adopted to evaluate the algorithms: satisfaction, i.e., percentage of users who have achieved their target MOS, minimum MOS and the computational cost.

One important metric of the system that will be used in the analyses is the coherence time, $T_{c}$, that is defined in [18] as the time duration over which the channel's response to a sinusoid has a correlation greater than 0.5 , and is given by

$$
T_{c}=\frac{0.423 c}{V \cdot f_{c}}
$$

in which $c=3.10^{8} \mathrm{~m} / \mathrm{s}$ is the speed of light, $f_{c}$ is the carrier frequency and $V$ is the relative speed between the UE and the BS. Considering the simulation parameters presented in Table I, we have $T_{c}=76 \mathrm{~ms}$.

\section{B. Numerical Results}

Firstly, we considered perfect knowledge of the CSI to evaluate the impact of the number of UEs on the satisfaction of the system, Figure 2, on the minimum MOS, Figure 3, and on the computational cost, Figure 4. 


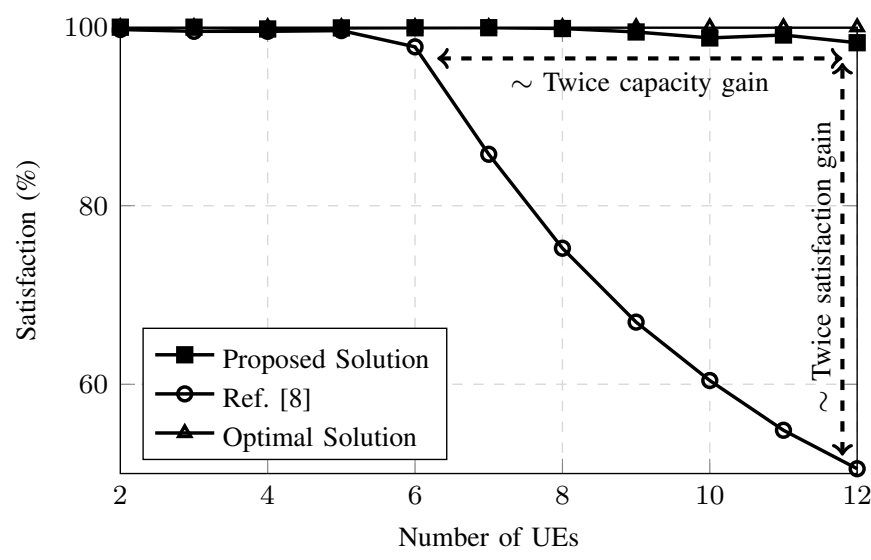

Fig. 2: Impact of the number of UEs on the satisfaction factor for target MOS equal to 3.5 .

In Figure 2, we can see that, on the one hand, [8] has a satisfaction lower than $100 \%$ in systems with more than 6 UEs. On the other hand, the proposed solution is able to support up to 12 UEs, while keeping almost $100 \%$ of the UEs satisfied, which means that the proposed solution is able to almost double the capacity of the system in terms of the number of UEs when compared to [8].

Notice also that not only the capacity can double, but also the satisfaction. For 12 UEs, while [8] achieves only $50 \%$ of satisfaction, our proposal still achieves satisfaction close to $100 \%$.

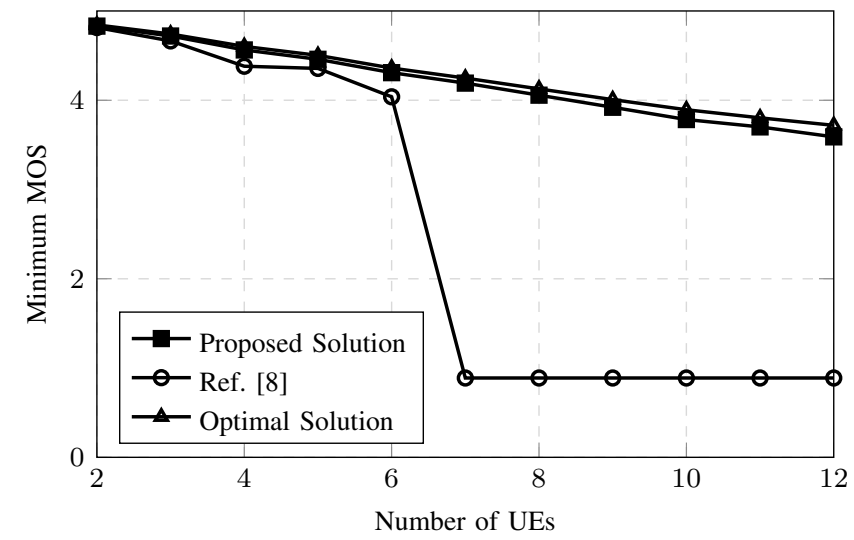

Fig. 3: Impact of the number of UEs on the Minimum MOS for target MOS equal to 3.5 .

Figure 3 presents the impact of the number of UEs on the minimum MOS. As in Figure 2, the proposed solution outperforms [8] and performs close to the optimal solution as well. The steep decrease observed between loads 6 UEs and 7 UEs highlights the scalability problem of [8]. It is not able to handle the cases in which the number of UEs is higher than the number of RBs. Our proposal solves this problem by excluding users already satisfied and giving the opportunity to users with low MOS to increase their satisfaction.

Figure 4 presents the impact of the number of UEs on the computational cost of the three analyzed algorithms. The mean time required by each algorithm to reach a solution was

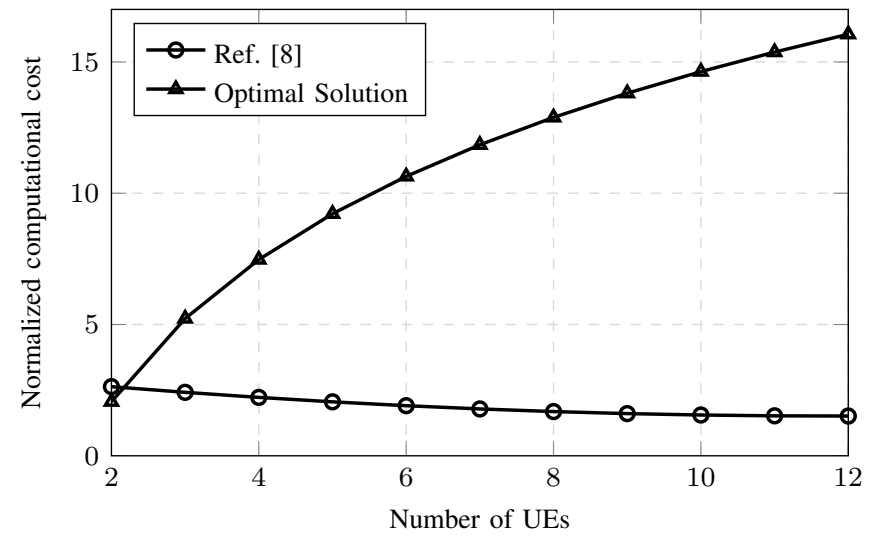

Fig. 4: Normalized computational cost for target MOS equal to 3.5.

normalized by the mean time required by our proposal. As one can see, the curve representing [8] is almost constant and has a low value, which means that the proposed scheduler and [8] have a similar computational cost. Since Ref. [8] has also a power allocation step, it takes a little bit more time to run. Concerning the optimal solution it has a logarithmic relationship with our proposal. Notice that, for 6 UEs, it takes more than $10 \mathrm{x}$ the time required by our proposal to run.

Figure 5 presents the impact on the satisfaction factor of changing UEs from service plan 1 (target MOS 3.5) to service plan 2 (target MOS 4.5), in a system with 6 UEs. As we can see, in the considered scenario, [8] is not even able to keep at least one UE in service plan 2, while keeping the satisfaction rate in the system higher than $90 \%$. In opposite, our proposal can keep at most 4 UEs with a high target MOS, while keeping the satisfaction close to the optimal value.

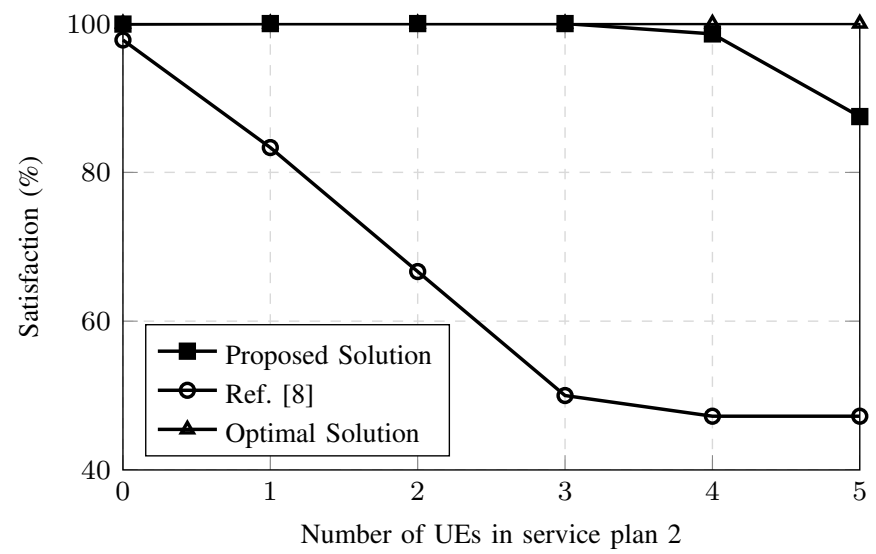

Fig. 5: Impact on the satisfaction factor of changing UEs from service plan 1 (target MOS 3.5) to service plan 2 (target MOS 4.5).

The last analyses focused on the delay of the CSI estimation. For this scenario, the optimal solution was not considered. Figures 6 and 7 present the impact of the delay, $\Delta t$, on the satisfaction and on the minimum MOS, respectively.

From Figures 6 and 7 we can see that the increasing of CSI estimation delay negatively impacts the algorithms performance only for $0<\Delta t<70 \mathrm{~ms}$. For $\Delta t>70$ 


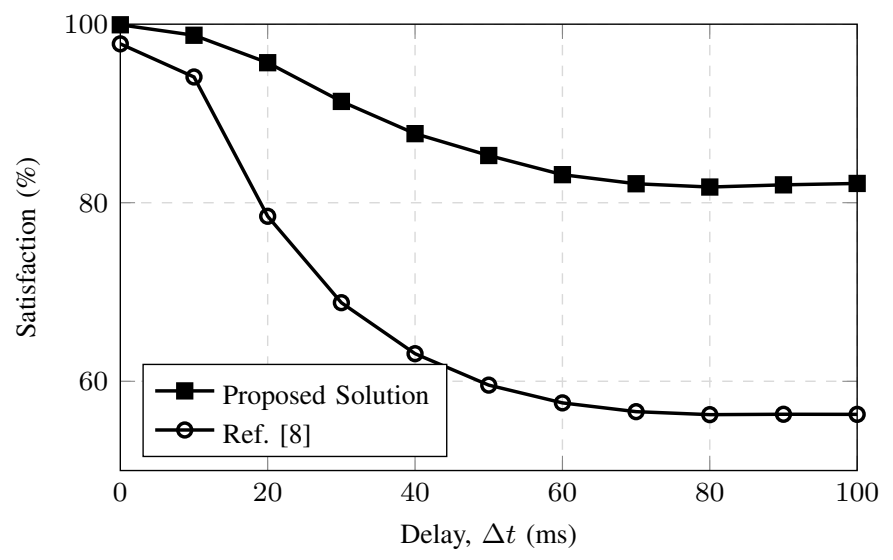

Fig. 6: Impact of the delay on the satisfaction factor for target MOS equal to 3.5 .

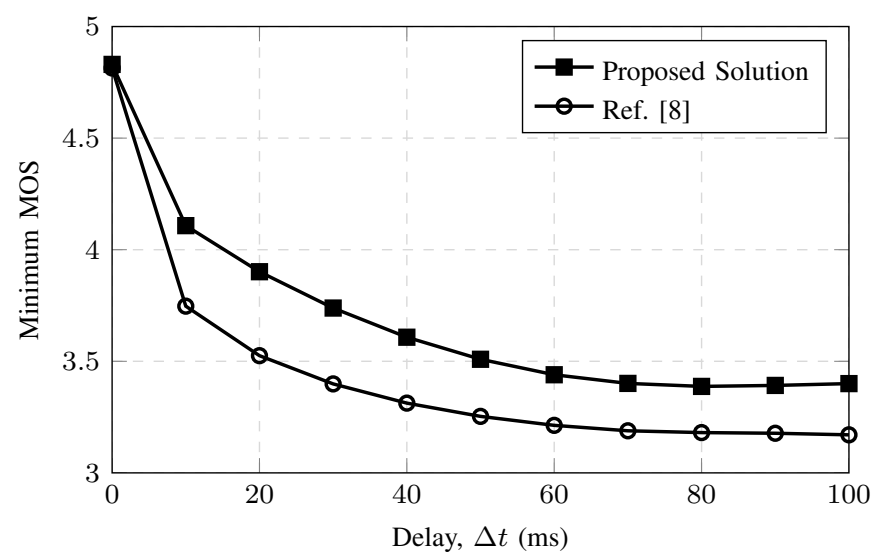

Fig. 7: Impact of the delay on the Minimum MOS for target MOS equal to 3.5 .

ms, approximately, the performance of the algorithms does not change significantly. This is due to the coherence time, $T_{0}=76 \mathrm{~ms}$. For $\Delta t>T_{0}$, the channel estimate received by the eNB, $\hat{h}_{u, k}[t]$, and the real channel measure, $h_{u, k}[t]$, are already almost uncorrelated. Therefore, the increase of $\Delta t$ does not imply in less information about the channel, which justifies the plateau for $\Delta t>T_{0}$.

Since our proposal considers the previous allocations, if a UE loses a packet due to the error in channel estimate, in the next TTI, it will be able to handle this situation. This results in a better performance compared to [8]. Notice that the proposed solution is able to keep more than $80 \%$ of the UEs satisfied, while [8] keeps fewer UEs satisfied.

\section{CONClusion ANd PeRspectives}

In this paper, we have presented a new QoE-aware scheduler aiming at maximizing the minimum MOS in a system subject to attaining at least a given number of satisfied users.

Different from the benchmark solution, the proposed scheduler takes into account all the data that the UEs have already received, and not only the data of a specific instant of time, as well as it excludes from scheduling process the UEs that are already satisfied.

As demonstrated, the proposed solution outperforms the benchmark solution, as well as it performs close to the optimal one. It improves the users' satisfaction and the minimum experienced MOS in the system, being able of doubling the capacity of the system in terms of number of users. It is also able to handle imperfections on the CSI estimation.

Due to the NP-Hardness of the studied problem, the application of soft computing techniques such as evolutionary algorithms and metaheuristics seem to be promising research areas. Future investigations might try to apply soft computing techniques in order to obtain near optimal solutions for a large number of RBs.

\section{ACKNOWLEDGMENT}

Victor F. Monteiro would like to acknowledge FUNCAP for its scholarship support.

\section{REFERENCES}

[1] V. F. Monteiro, D. A. Sousa, T. F. Maciel, F. R. M. Lima, and F. R. P. Cavalcanti, "Alocação de recursos em redes sem fio baseada na qualidade de experiência do usuário", in XXXIII Brazilian Telecommunications Symposium (SBrT'15), 2015.

[2] J. G. Andrews, S. Buzzi, W. Choi, S. V. Hanly, A. E. Lozano, A. C. K. Soong, and J. C. Zhang, "What will 5G be?", IEEE Journal on Selected Areas in Communications, vol. 32, pp. 1065-1082, 2014. DOI: 10.1109/ JSAC.2014.2328098.

[3] ITU, "Method for subjective determination of transmission quality", in ITU-T Recommendation, 1996, p. 800.

44] X. Chen, J.-N. Hwang, C.-N. Lee, and S.-I. Chen, "A near optima QoE-driven power allocation scheme for scalable video transmissions over MIMO systems", IEEE Journal of Selected Topics in Signal Processing, vol. 9, no. 1, pp. 76-88, Feb. 2015, ISSN: 1932-4553.

[5] J. Zheng, Y. Cai, Y. Liu, Y. Xu, B. Duan, and X. Shen, "Optimal power allocation and user scheduling in multicell networks: Base station cooperation using a game-theoretic approach", IEEE Transactions on Wireless Communications, vol. 13, no. 12, pp. 6928-6942, 2014, ISSN: 1536-1276.

[6] X. Deng, Q. Gao, S. Mohammed, L. Chen, F. Wang, and Z. Fei, "A QoE-driven resource allocation strategy for OFDM multiuser-multiservice system", in IEEE International Conference on Computer and Information Technology (CIT), 2014, pp. 351-355. DOI: 10.1109/CIT.2014.47.

[7] Y. H. Cho, H. Kim, S.-H. Lee, and H. S. Lee, "A QoE-aware proportional fair resource allocation for multi-cell OFDMA networks", IEEE Communications Letters, vol. 19, no. 1, pp. 82-85, 2015, ISSN: 1089-7798. DOI: 10.1109/LCOMM.2014.2367115.

[8] V. F. Monteiro, D. A. Sousa, T. F. Maciel, F. R. M. Lima E. B. Rodrigues, and F. R. P. Cavalcanti, "Radio resource allocation framework for quality of experience optimization in wireless networks", IEEE Network, vol. 29, no. 6, pp. 33-39, Nov. 2015. DOI: 10.1109/MNET.2015.7340422.

[9] 3GPP, "Physical layer aspects for evolved universal terrestrial radio access (UTRA)", Third Generation Partnership Project, TR 25.814 V7.1.0, Sep. 2006.

[10] — "Evolved universal terrestrial radio access (E-UTRA); further advancements for E-UTRA physical layer aspects", 3rd Generation Partnership Project (3GPP), TR 36.814, Mar. 2010. [Online]. Available: http://www.3gpp.org/ftp/Specs/html-info/36814.htm.

[11] G. Nemhauser and L. Wosley, Integer and Combinatorial Optimization. John Wiley \& Sons, 1999.

[12] T. M. Apostol, One-Variable Calculus with an Introduction to Linear Algebra, 2nd. Wiley, 1991.

[13] A. Cichocki, R. Zdunek, A. H. Phan, and S. ichi Amari, Nonnegative Matrix and Tensor Factorizations: Applications to Exploratory Multi-way Data Analysis and Blind Source Separation, 1st. Wiley, 2009. 
[14] F. Gunnarsson, M. Johansson, A Furuskar, M. Lundevall, A Simonsson, C. Tidestav, and M. Blomgren, "Downtilted base station antennas - a simulation model proposal and impact on HSPA and LTE performance", in Proceedings of the IEEE Vehicular Technology Conference (VTC), Sep. 2008, pp. 1-5. DOI: 10.1109/VETECF.2008. 49.

[15] 3GPP, "Deployment aspects", Third Generation Partnership Project, Tech. Rep. TR 25.943 V9.0.0, Dec. 2009.

[16] C. Mehlführer, M. Wrulich, J. C. Ikuno, D. Bosanska, and M. Rupp, "Simulating the long term evolution physical layer", in Proceedings of the European Signal Processing Conference, Glasgow, Scotland, Aug. 2009, pp. 1471-1478.

[17] J. Poncela, G. Gomez, A. Hierrezuelo, F. Lopez-Martinez, and M. Aamir, "Quality assessment in 3g/4g wireless networks", Wireless Personal Communications, vol. 76, no. 3, pp. 363-377, Mar. 2014. DOI: $10.1007 / \mathrm{s} 11277-014-1711-5$.

[18] B. Sklar, "Rayleigh fading channels in mobile digital communication systems part i: Characterization", IEEE Communications Magazine, vol. 35 , no. 7 , pp. $90-100,1997$, ISSN: 0163-6804. DOI: $10.1109 / 35$. 601747.

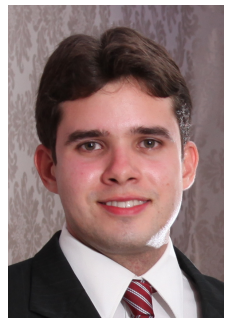

Victor Farias Monteiro received the double B.Sc. degree in General Engineering, from the École Centrale Lyon, France, and in Telecommunications Engineering (magna cum laude), from the Federal University of Ceará (UFC), Fortaleza, Brazil, in 2013. In 2015, he received the M.Sc. degree in Telecommunications Engineering from UFC. He is currently a Ph.D. student and researcher at the Wireless Telecom Research Group (GTEL), UFC, where he works in projects in cooperation with Ericsson Research. From 2010 to 2012, he took part, in France, of the Eiffel Excellence Scholarship Programme, established by the French Ministry of Foreign Affairs. In 2011, he was intern at Airbus, Toulouse, France. In 2012, he was intern at Gemalto, Paris, France, working on Machine-to-Machine technology for photovoltaic systems. His research interests include numerical optimization, $5 \mathrm{G}$ wireless communication networks and radio resource allocation algorithms for QoS/QoE provisioning.

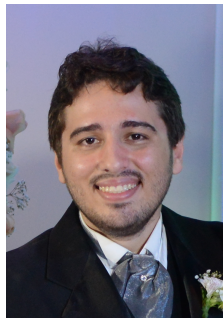

Diego Aguiar Sousa received the B.Sc. degree in Computer Engineering in University of Ceará (UFC), Sobral, Brazil, in 2011. In 2013, he received the M.Sc. degree in Telecommunications Engineering from the UFC, Fortaleza, Brazil. Since 2013, he has been a Ph.D. student and researcher at the Wireless Telecom Research Group (GTEL), UFC, participating of projects in a technical and scientific cooperation with Ericsson Research. Also, since 2013, he has been a Professor of the Federal Institute of Education, Science, and Technology of Ceará (IFCE), Acaraú, Brazil. His research interests include numerica optimization, 5G wireless communication networks, coordinated scheduling, radio resource allocation for QoS/QoE provisioning.

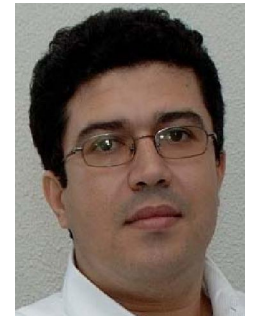

Tarcisio Ferreira Maciel received his B.Sc. and M.Sc. degrees in Electrical Engineering from the Federal University of Ceará (UFC) in 2002 and 2004, respectively, and his Dr.-Ing. degree from the Technische Universität Darmstadt (TUD), Germany, in 2008, also in Electrical Engineering. Since 2001, he has actively participated in several projects in a technical and scientific cooperation between Wireless Telecom Research Group (GTEL), UFC, and Ericsson Research. From 2005 to 2008, he was a research assistant with the Communications Engineering Laboratory, TUD Since 2008, he has been a member of the Post-Graduation Program in Teleinformatics Enginnering, UFC. In 2009, he was a Professor of computer engineering with UFC-Sobral and since 2010, he has been a Professor with the Center of Technology, UFC. His research interests include radio resource management, numerical optimization, and multiuser/multiantenna communications.

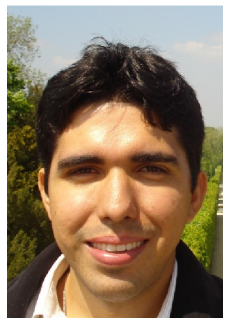

Francisco Rafael Marques Lima received the B.Sc. degree with honors in Electrical Engineering in 2005, and M.Sc. and D.Sc. degrees in Telecommunications Engineering from the Federal University of Ceará, Fortaleza, Brazil, in 2008 and 2012, respectively. In 2008, he has been in an internship at Ericsson Research in Luleå, Sweden, where he studied scheduling algorithms for LTE system. Since 2010, he has been a Professor of Computer Engineering Department of Federal University of Ceará, Sobral, Brazil. Prof. Lima is also a researcher at the Wireless Telecom Research Group (GTEL), Fortaleza Brazil, where he works in projects in cooperation with Ericsson Research. He has published several conference and journal articles as well as patents in the wireless telecommunications field. His research interests include radio resource allocation algorithms for QoS guarantees in scenarios with multiple services, resources, antennas and users.

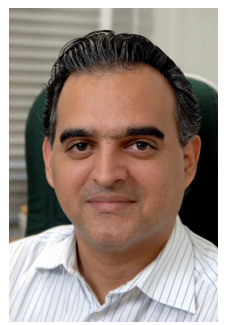

Francisco Rodrigo Porto Cavalcanti received the B.Sc. and M.Sc. degrees in Electrical Engineering from Federal University of Ceará (UFC), Fortaleza, Brazil, in 1994 and 1996, respectively, and the D.Sc. degree in Electrical Engineering from the State University of Campinas, São Paulo, Brazil, in 1999. Upon graduation, he joined the UFC, where he is currently an Associate Professor and holds the Wireless Communications Chair with the Department of Teleinformatics Engineering. In 2000, he founded and, since then has directed the Wireless Telecom Research Group (GTEL), which is a research laboratory based on Fortaleza, which focuses on the advancement of wireless telecommunications technologies. At GTEL, he manages a program of research projects in wireless communications sponsored by the Ericsson Innovation Center in Brazil and Ericsson Research in Sweden. Prof. Cavalcanti has produced a varied body of work including two edited books, conference and journal papers, international patents and computer software dealing with subjects such as radio resource allocation, cross-layer algorithms, quality of service provisioning, radio transceiver architectures, signal processing and project management. Prof. Cavalcanti is a distinguished researcher of the Brazilian Scientific and Technological Development Council for his technology development and innovation record. He also holds a Leadership and Management professional certificate from the Massachusetts Institute of Technology, Cambridge. 